\title{
Upgrade of the CMS muon trigger system in the barrel region
}

C. Battilana ${ }^{1}$, R. Carlin ${ }^{2}$, G. Codispoti ${ }^{1}$, M. Dallavalle ${ }^{1}$, J. Erö ${ }^{3}$, G. Flouris $^{4}$, C. Foudas $^{4}$, J. Fulcher ${ }^{5}$, L. Guiducci ${ }^{1}$, N. Loukas ${ }^{4}$, S. Mallios ${ }^{4}$, N. Manthos ${ }^{4}$, I. Papadopoulos $^{4}$, E. Paradas ${ }^{4}$, D. Rabady ${ }^{* 3}$, T. Reis ${ }^{5}$, H. Sakulin ${ }^{5}$, P. Sphicas ${ }^{5}$, A. Triossi $^{5}$, A. Venturi ${ }^{6}$, C.-E. Wulz ${ }^{3}$

on behalf of the CMS Collaboration

${ }^{1}$ University of Bologna

${ }^{2}$ University of Padua

${ }^{3}$ Institute of High Energy Physics Vienna (HEPHY)

${ }^{4}$ University of Ioannina

${ }^{5}$ European Organization for Nuclear Research (CERN)

${ }^{6}$ University of Pisa

E-mail: dinyarecern.ch

To maintain the excellent performance of the LHC during its Run-1 also in Run-2, the Level-1 Trigger of the Compact Muon Solenoid experiment underwent a significant upgrade. One part of this upgrade was the re-organisation of the muon trigger path from a subsystem-centric view in which hits in the drift tubes, the cathode strip chambers, and the resistive plate chambers were treated separately in dedicated track-finding systems, to one in which complementary detector systems for a given region (barrel, overlap, and endcap) are merged already at the track-finding level. This also required the development of a new system to sort as well as cancel-out the muon tracks found by each system.

An overview will be given of the new track-finder system for the barrel region, the Barrel Muon Track Finder (BMTF) as well as the cancel-out and sorting layer, the upgraded Global Muon Trigger $(\mu \mathrm{GMT})$. While the BMTF improves on the proven and well-tested algorithms used in the Drift Tube Track Finder during Run-1, the $\mu$ GMT is an almost complete re-development due to the re-organisation of the underlying systems from complementary track finders to regional track finders. Additionally, the $\mu \mathrm{GMT}$ can calculate a muon isolation using energy information that will be received from the calorimeter trigger in the future. This information is added to the muon objects forwarded to the Global Trigger.

Finally, first results of the muon trigger performance including the barrel region are shown. Both the trigger efficiency and the rate reduction show satisfactory performance, with improvements planned for the near future.

38th International Conference on High Energy Physics

3-10 August 2016

Chicago, USA

${ }^{*}$ Speaker. 


\section{Context}

The Level-1 Trigger of the Compact Muon Solenoid (CMS) experiment is responsible for reducing the event rate from the nominal Large Hadron Collider (LHC) bunch-crossing frequency of $40 \mathrm{MHz}$ to under $100 \mathrm{kHz}$.

The design philosophy of the Level-1 Trigger is to successively build physics objects in a pipelined system, combining local or regional features that were computed in earlier stages of the trigger until arriving at complete physics objects such as muons and jets. In later processing stages these objects are sorted and duplicates are removed or merged before sophisticated algorithms are applied that determine whether a readout signal shall be issued.

The legacy trigger system The original muon trigger $[1,2]$ processed hit information from each of the three muon detector systems (cathode strip chambers (CSC), drift tubes (DT), and resistive plate chambers (RPC)) separately to build complete muon tracks. The track objects reconstructed by the CSC track-finder (CSCTF), DT track-finder (DTTF), and RPC Pattern Comparator (PACT) were sorted at the outputs and the best four candidates of the CSCTF and DTTF as well as the best four candidates of the PACT in both the barrel and endcap regions were sent to the Global Muon Trigger (GMT) [3]. The GMT matched muons reconstructed by the RPC trigger as well as either CSCTF or DTTF according to flexible algorithms that improved the muon measurement by merging the matched tracks intelligently. Furthermore, muon tracks that were reconstructed twice in the overlap region between the barrel and endcap regions were found and the track with lower quality removed. In parallel the muons were sorted before the four highest-ranked muons were propagated to the Global Trigger (GT) that received both muon and calorimeter data and applied up to 128 algorithms, each of which could initiate a readout process.

The upgraded trigger system The LHC's expected peak instantaneous luminosity during Run-2 may exceed the original design specification by a factor two. To continue to provide an efficient trigger with good rate reduction a major upgrade was performed during the accelerator's Long Shutdown 1 that lasted from 2013 to 2014.

In the upgraded system [4], data from the muon detectors can be merged already in three new track-finder systems, the Barrel Muon Track Finder (BMTF), Overlap Muon Track Finder (OMTF), and Endcap Muon Track Finder (EMTF). Each track-finder system can process all available data from a given detector region and is split into 12 processors dedicated to a slice in azimuthal coverage. Each individual track-finder processor sends up to 3 muon candidates per LHC bunch crossing to the upgraded Global Muon Trigger ( $\mu \mathrm{GMT})$. The $\mu \mathrm{GMT}$ removes duplicates amongst these 108 muons, sorts them, and can apply isolation variables using energy information that will be received from the calorimeter trigger. It then sends the best eight muon candidates to the upgraded Global Trigger $(\mu \mathrm{GT})$ which can apply up to 512 algorithms in order to arrive at a readout decision.

\section{The upgraded barrel muon trigger path}

Barrel Muon Track Finder The BMTF [5] is responsible for reconstructing muon tracks in the barrel area $(|\eta|<0.83)$. It is logically split into twelve $30^{\circ}$ "wedges". Data coming from each 
wedge are treated by a dedicated track-finder processor, however hit information is shared between track-finder processors for neighbouring wedges in order to avoid inefficiencies at the overlaps.

A BMTF processor constructs the $\phi$ - and $\eta$-components of a track separately by extrapolating from given track segments to stations neighbouring in radial distance. Transverse momentum is determined by an algorithm exploiting both the difference in azimuthal coordinates of two track segments used to build the given track $\Delta \phi$, as well as the bending angle measured for the innermost used track segment, $\phi_{b}$. The value of $\Delta \phi$ is input to two look-up tables depending on whether a track is determined to be high- or low- $\mathrm{p}_{\mathrm{T}}$ as the same $\Delta \phi$ value can appear in high- or low- $\mathrm{p}_{\mathrm{T}}$ muon tracks. The bending angle is used both to distinguish between such high and low momentum muons, as well as to separately measure $\mathrm{p}_{\mathrm{T}}$ as it is well-correlated with transverse momentum for high- $\mathrm{p}_{\mathrm{T}}$ tracks. Currently only DT data is being used for this extrapolation, with commissioning for inclusion of RPC data ongoing. After a sorting stage the three best reconstructed muons from each sector are finally sent to the upgraded Global Muon Trigger.

Upgraded Global Muon Trigger The $\mu$ GMT [6] differs significantly from the legacy system, as it is not necessary to merge muons from the DT and CSC detectors with those delivered by the RPC system due to this information already being processed together at the track-finding level. In order to save latency, the $\mu \mathrm{GMT}$ integrates the formerly independent final sort and cancel-out layers of the track-finders. This means the $\mu \mathrm{GMT}$ is required to perform canceling-out of duplicates at each boundary between two track-finder processors. The cancel-out process works in parallel to a two-staged sorter that determines the best eight muons to forward to the $\mu \mathrm{GT}$ based on their reconstruction quality and transverse momentum. Finally, the $\mu$ GMT can compute the isolation of a muon based on the energy deposits in the calorimeter around the muon track at the interaction region. This functionality is scheduled to be commissioned in the future.

\section{Performance of the muon trigger}

The upgraded Level-1 Trigger has been used for CMS data taking from the beginning of 2016. An initial performance study was made, showing satisfactory performance with room for improvement by tuning the existing algorithms, as well as commissioning of the planned inclusion of RPC hits in both the BMTF and EMTF systems. Figure 1 shows the reconstruction efficiency of the Level-1 trigger for muons within a pseudo-rapidity range of $|\eta|<2.4$ with a Level-1 transverse momentum $\left(\mathrm{p}_{\mathrm{T}}\right)$ threshold of $22 \mathrm{GeV}$ plotted against the transverse momentum and the pseudorapidity of the reconstructed muon. A drop in efficiency discovered for muons with high transverse momentum could be attributed to a problem in the EMTF firmware and was fixed in August 2016.

The study was performed using the tag and probe technique on a dataset of events recorded using a single muon trigger in the 2016 run period until mid-July and considered the full detector coverage. The rate for the Level-1 single muon algorithm at an instantaneous luminosity of $6.7 \times 10^{33} \mathrm{~cm}^{-2} \mathrm{~s}^{-1}$ was measured to be $3.5 \mathrm{kHz}$ for a $22 \mathrm{GeV}$ threshold in muon $\mathrm{p}_{\mathrm{T}}$.

\section{Summary}

An overview of the upgraded CMS muon trigger in the barrel region was given. In the upgrade the formerly detector-centric track-finders were replaced by regional ones, requiring in turn 

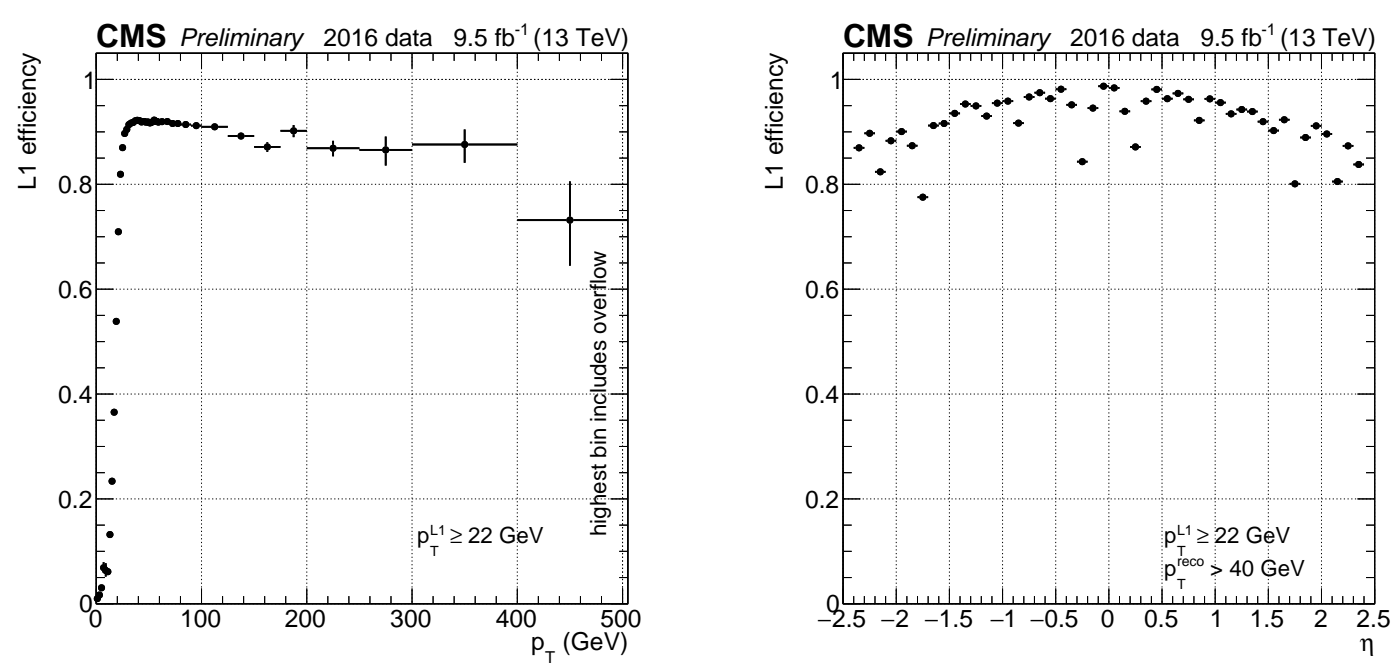

Figure 1: The Level-1 muon trigger efficiency at a $22 \mathrm{GeV}$ cut computed using tag and probe. The highest $\mathrm{p}_{\mathrm{T}}$ bin contains any muon with $\mathrm{p}_{\mathrm{T}}>400 \mathrm{GeV}$. The drop in efficiency at high $\mathrm{p}_{\mathrm{T}}$ was traced back to a firmware bug and was corrected in August 2016. The drops in $\eta$ are attributed to gaps in the muon system.

a replacement for the legacy Global Muon Trigger. The Barrel Muon Track Finder improves on an algorithm pioneered in the legacy system, building complete muon tracks by extrapolating between track segments from different muon stations. The upgraded Global Muon Trigger receives up to 108 muon candidates. These objects are examined for duplicates, sorted, and extrapolated to the interaction region in order to determine their isolation variable. The best eight muons are then sent to the upgraded Global Trigger together with a measure of their isolation.

Finally, a first study of the muon trigger performance was shown. A problem in the endcap for high momentum muons was found and solved which will improve the efficiency even further. Furthermore, inclusion of RPC data in the Barrel Muon Track Finder and Endcap Muon Track Finder will allow the systems to reconstruct tracks with higher quality and thus lead to enhanced performance.

\section{References}

[1] CMS Collaboration, CMS TriDAS project: Technical Design Report, Volume 1: The Trigger Systems, CMS-TDR-6-1

[2] CMS Collaboration, The CMS trigger system Submitted to JINST [physics.ins-det/1609.02366]

[3] H. Sakulin and A. Taurok, The Level-1 Global Muon Trigger for the CMS Experiment, CERN Conference Report CMS-CR-2003-040

[4] CMS Collaboration, CMS Technical Design Report for the Level-1 Trigger Upgrade, CMS-TDR-012

[5] J. Ero et al., The CMS Level-1 Trigger Barrel Track Finder, JINST 2016 1748-0221 11 C03038

[6] J. Lingemann et al., Upgrade of the CMS Global Muon Trigger, in proceedings of 19th IEEE-NPSS Real-Time conference 2014, Nara, Japan, 26 - 30 May 2014 pp.1104-1109, CMS-CR-2014-076 